\title{
A randomized clinical study to compare pre-emptive analgesic efficacy of clonidine and diclofenac sodium in patients undergoing major surgeries at a tertiary care hospital
}

\author{
Prachi Jain $^{1 *}$, Uma Bhosale ${ }^{2}$, Shashank Desai ${ }^{1}$
}

\begin{abstract}
${ }^{1}$ Department of Pharmacology, H. B. T. Medical College and R. N. Cooper Municipal General Hospital, Mumbai, Maharashtra, India

${ }^{2}$ Department of Pharmacology, Smt. Kashibai Navale Medical College and General Hospital, Mumbai, Maharashtra, India
\end{abstract}

Received: 14 October 2020

Revised: 11 December 2020

Accepted: 15 December 2020

*Correspondence:

Dr. Prachi Jain,

Email: jain1993prachi@gmail.com

Copyright: ( $)$ the author(s), publisher and licensee Medip Academy. This is an open-access article distributed under the terms of the Creative Commons Attribution Non-Commercial License, which permits unrestricted non-commercial use, distribution, and reproduction in any medium, provided the original work is properly cited.

\begin{abstract}
Background: Pre-emptive analgesia is a method to achieve analgesia even before exposure to a noxious stimulus The purpose of pre-emptive analgesia is to reduce pain caused due to surgical incision triggered inflammatory mechanism activation; and to ensure a good post-operative pain control so that there is no development of chronic pain. Clonidine is an $\alpha 2$ adrenergic agonist thus it decreases the sympathetic outflow, while Diclofenac is a non-steroidal antiinflammatory agent. Our literature review showed that both clonidine $(150 \mu \mathrm{g})$ and diclofenac are efficacious preemptive analgesics. Literature review also revealed that there is no study available to compare pre-emptive analgesic efficacy as well as safety of oral clonidine with diclofenac, therefore present study was designed to compare pre emptive analgesic efficacy of these two drugs.

Methods: This randomized clinical study included 100 patients from surgical departments, of either sex, between 18 to 70 years age and of American society of anesthesiologists (ASA) I/II grade. Patients were randomly allocated to two groups and received either of the treatments 30 minutes prior to induction of anesthesia. Pain scores were recorded using visual analog scale, facial rating scale and behavioral rating scale at awakening and at 1, 2, 4, 6 and 24 hours. Postoperative analgesic requirement over 24 hours was recorded. Data were analyzed using OpenEpi statistical softwares.

Results: Significantly lower pain scores were observed in clonidine group as compared to Diclofenac at 4 and 6 hours $(\mathrm{p}<0.05)$ on all the pain scales. Clonidine group also required less postoperative analgesic as compared to diclofenac $(\mathrm{p}<0.05)$.

Conclusions: Study results are strongly suggestive of greater pre-emptive analgesic efficacy of clonidine over diclofenac in major surgeries done under spinal anesthesia.
\end{abstract}

Keywords: Pre-emptive analgesia, Clonidine, Diclofenac, VAS, Aldrete's scale

\section{INTRODUCTION}

Major surgery is any invasive operative procedure in which a more extensive resection is performed; they are usually done under spinal anesthesia. Spinal anesthesia is an economical and simple technique but it comes with disadvantages like limited duration of analgesia leading to post-operative pain. ${ }^{1}$ It is important to achieve a better post-operative analgesia for inhibiting autonomic and somatic reflexes that arise in response to pain and restore 
function by allowing the patient to cough, breathe more easily. $^{2}$

Pre-emptive analgesia is an antinociceptive treatment which helps to develop premature analgesia even before exposure to a noxious stimulus. The purpose of preemptive analgesia is to reduce pain caused due to surgical incision triggered inflammatory mechanism activation; and to ensure a good post-operative pain control so that there is no development of chronic pain. ${ }^{3}$

The concept of preemptive analgesia has now become popular and some drugs such as opioid, local anesthetics, and nonsteroidal anti-inflammatory drugs have been demonstrated for their preemptive effect in previous studies. ${ }^{4}$ Various modalities have been tried but sideeffects produced by them limits their use.

Clonidine is an $\alpha 2$ adrenergic agonist and since $\alpha 2$ has an inhibitory effect on descending pain pathway, it decreases the sympathetic outflow in brain and spinal cord. Owing to this, it produces effects including sedation, analgesia, hypotension, and bradycardia. There is wide use of clonidine through various routes including intrathecal or intravenous administration in order to prolong the duration of spinal anesthesia without any adverse effects. ${ }^{5,6}$ Our literature review showed that clonidine $(150 \mu \mathrm{g})$ is an efficacious pre emptive analgesic and can be used safely along with spinal anesthesia. ${ }^{7}$ Diclofenac is a non-steroidal anti-inflammatory agent and is an established pre-emptive analgesic. ${ }^{8}$ Literature review also revealed that there is no study available to compare pre-emptive analgesic efficacy as well as safety of oral clonidine with diclofenac.

\section{Aim and objectives}

Aim and objectives of the study were: to evaluate the preemptive analgesic efficacy of single dose clonidine in the patients undergoing major surgeries, to compare the preemptive analgesic efficacy of clonidine and diclofenac sodium, and to estimate and compare the total postoperative analgesic consumption and post anaesthetic care unit (PACU) stay in both the groups.

\section{METHODS}

\section{Study type}

A prospective randomized study was conducted.

\section{Study place}

The study was conducted at a tertiary care hospital and medical college in Pune

\section{Study period}

The duration of the study was from December 2017 to June 2019.

\section{Inclusion criteria}

It included 100 patients, of either sex, between 18 to 70 years of age, having American society of anesthesiologists (ASA) grade I and II, from departments of surgery, obstetrics and gynecology and orthopedics undergoing major surgeries.

\section{Exclusion criteria}

Patients with diabetic complications and other diseases like congestive cardiac failure, arrhythmias, ischemic heart disease, uncontrolled hypertension, and stroke; cancer, renal, hepatic disease, bleeding diathesis, allergic to the study drugs pregnant or lactating females and those who were simultaneously involved in other trials, not willing to give consent were excluded from study.

\section{Procedure}

All patients were assessed preoperatively the day before surgery. Study protocol was explained. Subjects were randomly assigned to either of the two groups using a computer-generated table of random numbers to receive medications. With 50 subjects in each group, group A was given clonidine (oral $100 \mu \mathrm{g}$ tablet) 30 minutes prior to surgery and group B was given diclofenac (oral $100 \mathrm{mg}$ tablet) 30 minutes prior to surgery. ${ }^{9,10}$ Premedication with injection (inj) ondansetron (4 mg intravenously) and inj glycopyrrolate $0.04 \mathrm{mg} / \mathrm{kg}$ intravenous (i.v.) followed by induction of spinal anesthesia was done as per standard protocol. After completion of surgery, patients were transferred to post anesthetic care unit and from where they were transferred to their respective wards. Baseline and post-operative vital parameters such as pulse rate, respiratory rate, mean arterial pressures and saturation of peripheral oxygen $\left(\mathrm{SpO}_{2}\right)$ were recorded. Postoperative level of analgesia was noted on visual analog scale (VAS), facial rating scale (FRS) and behavioral rating scale (BRS) at 1, 2, 4, 6 and 24 hours. Rescue analgesic (diclofenac 75 $\mathrm{mg}$ ) was given if patient complained of pain or pain score was more than 4. Post-operative analgesic requirement for 24 hours was recorded. Duration of postoperative anesthesia care unit (PACU) stay was recorded by using modified Aldrete's scoring system; patients with score $>9$ were shifted to the ward.

\section{Ethical approval}

The protocol of study was approved by institutional ethics committee and written informed consent was obtained from each participant.

\section{Statistical analysis}

Sample size calculation was done by using OpenEpi statistical software with $95 \%$ confidence level, $80 \%$ power. Parametric data was analyzed using unpaired t test. Nonparametric data was analysed using Mann Whitney U test. Data was analyzed by using Open Epi statistical 
software. Significance level of 0.05 was considered as statistically significant.

\section{RESULTS}

In the present study entitled "A randomized clinical study to compare pre-emptive analgesic efficacy of clonidine and diclofenac sodium in patients undergoing major surgeries at a tertiary care hospital", was conducted after taking permission from institutional ethics committee (IEC). Total 100 patients fulfilling selection criteria were enrolled and results were as follows.

As presented in Table 1, the mean age of group A was $50.08 \pm 8.48$ years and mean age of group B was $50.22 \pm 9.18$ years. There were 30 males and 20 females in group A while 31 males and 19 females in group B.

As per the study protocol, vital parameters were observed, and no significant differences were found in both the groups as shown in Table 2.

When compared between two groups, it was found that pain scores on VAS, FRS, and BRS were statistically significant at 4 and 6 hours. The incidence of nausea and vomiting was 4 and 6 in clonidine and diclofenac groups, respectively (Table 3 ).

First rescue analgesia was required earlier in diclofenac group as compared to clonidine group. Frequency of rescue analgesic administration was higher in diclofenac group as compared to clonidine group. Both time and frequency of rescue analgesic administration showed statistical significance between the two groups. The present study showed that total diclofenac requirements were significantly less in the clonidine group. The PACU stay was the less in the clonidine as compared to diclofenac group and this difference between the 2 groups based on the PACU stay was statistically highly significant as shown in Table 4.

Table 1: Demographic profile $(n=100)$.

\begin{tabular}{|c|c|c|c|}
\hline $\begin{array}{l}\text { Characteristics } \\
(n=100)\end{array}$ & $\begin{array}{l}\text { Group } \\
\text { A }\end{array}$ & $\begin{array}{l}\text { Grou } \\
\text { p B }\end{array}$ & $\begin{array}{l}\mathbf{P} \\
\text { value }\end{array}$ \\
\hline Age in years $($ mean \pm SD) & $\begin{array}{l}50.08 \pm \\
8.48\end{array}$ & $\begin{array}{l}50.22 \\
\pm 9.18\end{array}$ & 0.74 \\
\hline Male: female & $30: 20$ & $31: 19$ & $\mathrm{NS}^{*}$ \\
\hline \multicolumn{4}{|l|}{ Type of operation } \\
\hline Abdominal hysterectomy & 3 & 3 & \multirow{8}{*}{ NS* } \\
\hline Open cholecystectomy & 2 & 3 & \\
\hline Total hip replacement & 3 & 4 & \\
\hline Vaginal hysterectomy & 9 & 10 & \\
\hline Amputation & 6 & 4 & \\
\hline Total knee replacement & 2 & 4 & \\
\hline Appendicectomy & 22 & 20 & \\
\hline Varicose veins & 3 & 2 & \\
\hline
\end{tabular}

Unpaired t test used for inter-group comparison; $*$ NS-statistically non-significant

Table 2: Vital parameters.

\begin{tabular}{|llllllrl|}
\hline Parameters & Baseline & Awakening & 1 hour & 2 hour & 4 hour & 6 hour \\
\hline Group A & & & & & & \\
\hline Pulse rate & $84.04 \pm 7.27$ & $63.52 \pm 3.96$ & $66.34 \pm 1.89$ & $70.28 \pm 2.30$ & $78.18 \pm 5.05$ & $84.38 \pm 6.78$ \\
\hline Respiratory rate & $14.14 \pm 1.37$ & $15.18 \pm 1.98$ & $16.1 \pm 1.67$ & $15.26 \pm 1.19$ & $15.02 \pm 1.27$ & $15.66 \pm 1.45$ \\
\hline SpO2 & $99.14 \pm 0.83$ & $98.76 \pm 0.87$ & $99.16 \pm 0.74$ & $98.88 \pm 0.82$ & $98.90 \pm 0.81$ & $98.98 \pm 0.82$ \\
\hline Mean arterial pressure & $86.78 \pm 4.80$ & $67.88 \pm 2.31$ & $69.28 \pm 2.01$ & $71.32 \pm 2.39$ & $77.82 \pm 4.10$ & $86.10 \pm 4.25$ \\
\hline Group B & & & & & \\
\hline Pulse rate & $84.54 \pm 8.26$ & $63.90 \pm 5.16$ & $66.82 \pm 4.52$ & $69.80 \pm 4.02$ & $78.82 \pm 5.86$ & $85.34 \pm 7.38$ \\
\hline Respiratory rate & $14.34 \pm 1.79$ & $15.46 \pm 1.88$ & $16.34 \pm 1.60$ & $15.46 \pm 1.43$ & $15.28 \pm 1.59$ & $16.02 \pm 1.62$ \\
\hline SpO2 & $99.06 \pm 0.79$ & $98.92 \pm 0.80$ & $99.06 \pm 0.77$ & $99.02 \pm 0.84$ & $99.06 \pm 0.82$ & $99.14 \pm 0.83$ \\
\hline Mean arterial pressure & $86.84 \pm 5.37$ & $68.32 \pm 1.87$ & $70.08 \pm 2.14$ & $72.14 \pm 2.0$ & $77.18 \pm 2.44$ & $86.70 \pm 4.64$ \\
\hline
\end{tabular}

Table 3: Pain scores.

\begin{tabular}{|c|c|c|c|c|c|c|c|}
\hline Groups & Awakening & 1 hour & 2 hour & 4 hour & 6 hour & 24 hour & ADRs \\
\hline \multicolumn{8}{|c|}{ Group A } \\
\hline VAS & $1.02 \pm 0.55$ & $0.98 \pm 0.62$ & $0.66 \pm 0.69$ & $3.22 \pm 0.93$ & $3.36 \pm 1.26$ & $5.02 \pm 0.74$ & \multirow{3}{*}{ Nausea/vomiting-4 } \\
\hline FRS & $1.03 \pm 0.53$ & $1 \pm 0.60$ & $0.72 \pm 0.70$ & $3.16 \pm 0.98$ & $3.24 \pm 1.27$ & $4.96 \pm 0.78$ & \\
\hline BRS & $1.06 \pm 0.55$ & $1.04 \pm 0.60$ & $0.74 \pm 0.69$ & $3.24 \pm 0.98$ & $3.28 \pm 1.20$ & $4.90 \pm 0.76$ & \\
\hline \multicolumn{8}{|l|}{ Group B } \\
\hline VAS & $1.04 \pm 0.60$ & $0.96 \pm 0.57$ & $0.8 \pm 0.67$ & $3.86 \pm 1.73$ & $3.98 \pm 1.62$ & $5.18 \pm 0.83$ & \multirow{3}{*}{ Nausea/ vomiting-6 } \\
\hline FRS & $1.04 \pm 0.60$ & $0.96 \pm 0.57$ & $0.8 \pm 0.67$ & $3.88 \pm 1.75$ & $3.90 \pm 1.54$ & $5.06 \pm 0.82$ & \\
\hline BRS & $1.05 \pm 0.60$ & $0.96 \pm 0.57$ & $0.8 \pm 0.67$ & $3.94 \pm 1.67$ & $3.90 \pm 1.47$ & $5 \pm 0.81$ & \\
\hline
\end{tabular}


Table 4: Time to first rescue analgesia and frequency of rescue analgesia.

\begin{tabular}{|llll|}
\hline Parameter & Group A (mean \pm SD) & Group B (mean \pm SD) & P value \\
\hline Time to $\mathbf{1}^{\text {st }}$ rescue analgesia (in minutes) & $393 \pm 119.93$ & $255.4 \pm 54.78$ & $<0.0001^{*}$ \\
\hline Frequency of rescue analgesia & $1.34 \pm 0.48$ & $2.02 \pm 0.62$ & $<0.0001^{*}$ \\
\hline Analgesic needed in 24 hours (in mg) & 5025 & 7575 & $<0.0001^{*}$ \\
\hline Per patient requirement, median (IQR); in mg & $75(75-150)$ & $150(150-225)$ & $<0.0001^{*}$ \\
\hline PACU stay (in minutes) & 61 & 82 & $<0.0001^{*}$ \\
\hline
\end{tabular}

$* \mathrm{p}<0.0001=$ statistically significant; unpaired $\mathrm{t}$ test used for inter-group.

\section{DISCUSSION}

Preemptive analgesia is a treatment that is intended to reduce the physiological consequences of nociceptive transmission provoked by the procedure. It is administered before the surgical procedure. Due to this-protective effect on the pain pathways, preemptive analgesia has the potential to be more effective than a similar analgesic treatment initiated after surgery. Consequently, it helps to reduce immediate postoperative pain and to prevent development of chronic pain. ${ }^{5}$

The demographic data was comparable between the two groups. No statistically significant differences were found between the groups with respect to age, sex distribution, type of surgery and duration of surgery. This allows us to evenly compare the results obtained. The mean baseline heart rate and mean arterial pressure were comparable between two groups $(p>0.05)$. There was no significant change in hemodynamic parameters in both the groups and the difference in mean heart rate and mean arterial pressure between both the groups were statistically insignificant preoperatively and postoperatively $(p>0.05)$. There was no significant change within the groups at different time intervals. According to a study, in patients undergoing laparoscopic cholecystectomy, premedication with oral clonidine $150 \mu \mathrm{g}$ results in improved perioperative hemodynamic stability when compared to a placebo. ${ }^{11}$ Few studies have reported that clonidine increases perioperative circulatory stability in patients undergoing laparoscopic cholecystectomy and potentiate parasympathetic nervous system. ${ }^{12,13}$ In our study, no statistical significance is suggestive of hemodynamic stability with both clonidine and diclofenac. There was no significant change in respiratory rate and $\mathrm{SpO}_{2}$ between the two groups and the difference in mean respiratory rate and $\mathrm{SpO}_{2}$ of patients was statistically insignificant preoperatively and postoperatively both between and within groups ( $p$ value $>0.05$ ). According to a study, same dose of $100 \mu \mathrm{g}$ Clonidine does not cause respiratory depression and produces adequate analgesia. ${ }^{8}$

For postoperative pain assessment VAS, BRS and FRS were used. The VAS, BRS and FRS scores were lower in group A than group B at all set time points, with the difference reaching statistical significance at 4 and 6 hours. In a meta-analysis of randomized trials, patients receiving pre-emptive local anesthetic wound infiltration and nonsteroidal anti-inflammatory drug administration experienced a decrease in analgesic consumption, but no decrease in postoperative pain scores. Pre emptive epidural analgesia did show a decrease in pain scores as well as analgesic consumption. ${ }^{14}$ In another study, the effects of diclofenac, paracetamol, and their combination on pain after tonsillectomy were compared. Two drugs combination caused less pain after surgery but there was no significant difference between the effects of diclofenac and paracetamol. ${ }^{15}$ Some other studies also investigated the effect of diclofenac on pain after tonsillectomy surgery or other surgeries, all of which indicated the pain-relieving effect of diclofenac and that less opioid consumption was required after surgery. 16,17

First rescue analgesia was required earlier in diclofenac group as compared to clonidine group. Frequency of rescue analgesic administration was higher in diclofenac group as compared to diclofenac group. Both time and frequency of rescue analgesic administration showed statistical significance between the two groups. After applying student's t-test to both the groups at different time intervals, we found the difference between total rescue analgesic requirements to be statistically highly significant. The clonidine group required less rescue analgesic as compared to diclofenac group. Oral/ injectable diclofenac was used as a rescue analgesic. This finding is like earlier studies as mentioned above. According to a study, postoperative analgesic requirements were reduced when oral clonidine $150 \mu \mathrm{g}$ was administered as a pre-medicant in patients undergoing laparoscopic cholecystectomy. ${ }^{11}$ The present study showed that total diclofenac requirements were significantly less in the clonidine group. NSAIDs are commonly used analgesics in surgical procedures for decreasing pain and opioid requirements. ${ }^{18}$ They are well established, effective, and inexpensive; however, inadequate analgesia and adverse renal, gastrointestinal, and hemostatic effects may limit their use in some groups of patients. ${ }^{19,20}$ This finding is like earlier studies as mentioned above. The PACU stay was the less in the clonidine as compared to diclofenac group and this difference between the 2 groups based on the PACU stay was highly significant. The side effects we observed in our study were the postoperative nausea and vomiting (PONV) where 4 patients of clonidine group and 6 patients of diclofenac group were affected. The difference between the post-operative vomiting episodes in the two groups is not statistically significant. All the subjects who suffered nausea and vomiting were treated by tablet ondansetron 4 mg orally. Factors like age and gender of patient, obesity, technique of anesthesia employed, presence of postoperative pain, use of opioids for pain management and 
elective surgical procedures also influence the incidence of PONV.

The limitation of this study is that the pre-emptive analgesic efficacy was studied only in patients operated under spinal anesthesia; patients operated under general anesthesia as well need to be studied. Furthermore, we have evaluated preemptive analgesic efficacy of single oral dose of $100 \mu \mathrm{g}$ clonidine; the single highest safe dose needs to be evaluated to offer maximal post-operative pain control through practicing pre-emptive analgesia.

\section{CONCLUSION}

This study revealed that clonidine has better pre-emptive analgesic efficacy and safety as compared to diclofenac based on pain scores, frequency of rescue analgesic requirements, duration of PACU stay and adverse events.

\section{Funding: No funding sources}

Conflict of interest: None declared

Ethical approval: The study was approved by the Institutional Ethics Committee

\section{REFERENCES}

1. Lovich-Sapola J, Smith CE, Brandt CP. Postoperative Pain Control. Surg Clin North Am. 2015;95(2):30118.

2. Vasure R, Ashahiya ID, Mahendra R, Narang N, Bansal RK. Comparison of Effect of Adding Intrathecal Magnesium Sulfate to Bupivacaine Alone and Bupivacaine-Fentanyl Combination during Lower Limb Orthopedic Surgery. Int J Sci Study. 2016;3(10):141-6.

3. Kolarkar P, Badwaik G, Watve A, Agarkar S, Kalbande J, Giri A, et al. Pre-emptive oral clonidine for immediate postoperative pain in surgeries under sub-arachnoid block. J Evol Med Dent Sci. 2014;3:11913-21.

4. Kashefi P, Honarmand A, Safavi M. Effects of preemptive analgesia with celecoxib oral acetaminophen on postoperative pain relief following lower extremity orthopedic surgery. Adv Biomed Res. 2012;1:66.

5. Woolf CJ, Chong MS. Preemptive analgesia--treating postoperative pain by preventing the establishment of central sensitization. Anesth Analg. 1993;77(2):36279.

6. Kanazi GE, Aouad MT, Jabbour-Khoury SI, Al Jazzar MD, Alameddine MM, Al-Yaman R, et al. Effect of low-dose dexmedetomidine or clonidine on the characteristics of bupivacaine spinal block. Acta Anaesthesiol Scand. 2006;50(2):222-7.

7. Beniwal S, Chaudhary S. Pre-emptive oral pregabalin versus clonidine for immediate post-operative pain in surgeries under sub arachnoid block. Available at: www.wjpmr.com. Accessed on 20 July 2020.
8. Shah P, Bhosale UA, Gupta A, Yegnanarayan R, Sardesai S. A randomized double-blind placebocontrolled study to compare preemptive analgesic efficacy of novel antiepileptic agent lamotrigine in patients undergoing major surgeries. North Am J Med Sci. 2016;8(2):93.

9. Gupta K, Singh I, Singh VP, Gupta P, Tiwari V. Preemptive analgesia of oral clonidine during subarachnoid block for laparoscopic gynecological procedures: A prospective study. Anesth Essays Res. 2014;8(2):187.

10. Zacharias M, Hunter K, Baker B. Effectiveness of preoperative analgesics on postoperative dental pain: A study. Anesth Prog. 1996;43:92-6.

11. Singh S, Arora K. Effect of oral clonidine premedication on perioperative haemodynamic response and postoperative analgesic requirement for patients undergoing laparoscopic cholecystectomy. Indian J Anaesth. 2011;55(1):26-30.

12. Hayashi Y, Maze M. Alpha 2 adrenoceptor agonists and anaesthesia. Br J Anaesth. 1993;71(1):108-18.

13. Sung CS, Lin SH, Chan KH, Chang WK, Chow LH, Lee TY. Effect of oral clonidine premedication on perioperative hemodynamic response and postoperative analgesic requirement for patients undergoing laparoscopic cholecystectomy. Acta Anaesthesiol Sin. 2000;38(1):23-9.

14. Ong CK-S, Lirk P, Seymour RA, Jenkins BJ. The efficacy of preemptive analgesia for acute postoperative pain management: a meta-analysis. Anesth Analg. 2005;100(3):757-73.

15. Hiller A, Silvanto M, Savolainen S, Tarkkila P. Propacetamol and diclofenac alone and in combination for analgesia after elective tonsillectomy. Acta Anaesthesiol Scand. 2004;48(9):1185-9.

16. Romsing J, Ostergaard D, Drozdziewicz D, Schultz P, Ravn G. Diclofenac or acetaminophen for analgesia in paediatric tonsillectomy outpatients. Acta Anaesthesiol Scand. 2000;44(3):291-5.

17. Schmidt A, Bjorkman S, Akeson J. Preoperative rectal diclofenac versus paracetamol for tonsillectomy: effects on pain and blood loss. Acta Anaesthesiol Scand. 2001;45(1):48-52.

18. White PF. The changing role of non-opioid analgesic techniques in the management of postoperative pain. Anesth Analg. 2005;101(5):5-22.

19. Schafer AI. Effects of nonsteroidal antiinflammatory drugs on platelet function and systemic hemostasis. J Clin Pharmacol. 1995;35(3):209-19.

20. Bjorkman D. Nonsteroidal anti-inflammatory drugassociated toxicity of the liver, lower gastrointestinal tract, and esophagus. Am J Med. 1998;105(5A):17-21.

Cite this article as: Jain P, Bhosale U, Desai S. A randomized clinical study to compare pre-emptive analgesic efficacy of clonidine and diclofenac sodium in patients undergoing major surgeries at a tertiary care hospital. Int J Basic Clin Pharmacol 2021:10:44-8. 